Research Article

\title{
Study of Prothrombotic Markers in COPD
}

\author{
Mradul Kumar Daga', Naresh Kumar ${ }^{2}$, Govind Mawari ${ }^{3}$, Shashank Singh ${ }^{4}$,
}

Soumya Jagannath Mahapatra ${ }^{5}$, Maryam Hussain ${ }^{6}$, Manish K Jha $^{7}$

${ }^{1}$ Director Professor, ${ }^{4}$ Senior Resident, ${ }^{5}$ Resident, ${ }^{6}$ Department of Medicine, Maulana Azad Medical College, New Delhi, India. ${ }^{2}$ Professor of Medicine \& Head, Pulmonary Medicine, Maulana Azad Medical College, New Delhi, India.

${ }^{3}$ Scientist - C, ${ }^{7}$ Research Associate, Centre for Occupational and Environmental Health, Maulana Azad Medical College, New Delhi, India.

DOI: https://doi.org/10.24321/2349.7181.202014

\section{I $\quad \mathbf{N} \quad \mathbf{F} \quad \mathbf{O}$}

\section{Corresponding Author:}

Mradul K Daga, Department of Medicine, Maulana Azad Medical College, New Delhi, India. E-mail Id:

drmraduldaga@gmail.com

Orcid Id:

https://orcid.org/0000-0001-7774-7602

How to cite this article:

Daga MK, Kumar N, Singh S, Mawari G, Mahapatra SJ, Jha Mk et al. Study of Prothrombotic Markers in COPD. J Adv Res Med 2020; 7(4): 1-6.

Date of Submission: 2020-12-03

Date of Acceptance: 2020-12-19

\section{$\begin{array}{llllllll}\mathbf{A} & \mathbf{B} & \mathbf{S} & \mathbf{T} & \mathbf{R} & \mathbf{A} & \mathbf{C} & \mathbf{T}\end{array}$}

Background: Chronic Obstructive Pulmonary Disease (COPD) is chronic inflammatory condition that is known to be a prothrombotic state.

Objective: To study the prothrombotic markers and coagulation profile in patients of Stable COPD and during its exacerbation and to compare these parameters in stable phase of disease and during exacerbation.

Materials and Method: A cross sectional study in which 30 patients of COPD with exacerbation and 30 patients with stable COPD were recruited. Patients having acquired thrombotic risk factors like diabetes, hypertension, coronary artery disease, chronic kidney disease, chronic liver disease, malignancy \& sepsis or any immunocompromised condition along with those who were on anticoagulant therapy, statins or antihypertensive medications were excluded from the study. Levels of prothrombotic markers (von Willebrand Factor antigen, D-dimer, fibrogen) and Caogulation profile (Prothrombin time and activated Partial Thromboplastin Time) were estimated in both the groups and repeated after stabilization of exacerbation phase also. There parameters were then statistically analyzed.

Observation and Results: A generalized prothrombotic state was observed in exacerbation phase of COPD as compared to stable phase of COPD. Furthermore, all the parameters including fibrinogen, vWF, PT and aPTT showed persistent declining trend from exacerbation towards stabilization.

Conclusion: COPD with acute exacerbation is a state of augmented prothrombotic state. On stabilization of acute exacerbation with treatment, coagulation profile came to a level lower than that of patients in stable phase of COPD. In exacerbation phase of COPD vWF values were significantly elevated than in stable phase.

Keywords: COPD, Prothrombotic Markers, Fibrinogen, Von Willebrand Factor, Acute Exacerbation of COPD 


\section{Introduction}

Chronic Obstructive Pulmonary Disease (COPD), a very common disease involving partial reversibility of airflow limitation, has at its core the continuing inflammation of respiratory system as well as at systemic level. ${ }^{1}$ Various extra pulmonary or systemic effects of COPD can be attributed to this persistent inflammatory state. ${ }^{2}$ Exacerbations, a part of natural disease process of COPD, represent amplification of this inflammatory response which is associated with increased concentrations of certain inflammatory mediators i.e., TNF- $\alpha$, LT $\beta-4$ \& IL-8, etc.

Increased pulse wave velocity, a surrogate marker for central arterial stiffness, endothelial dysfunction \& atherosclerosis correlate with reduced $\mathrm{FEV}_{1}{ }^{3}$ This consequently leads to crowning of CAD as one of the leading cause of death in COPD patients. ${ }^{4}$ Looking forward the association of COPD \& atherothrombosis, four pathological factors are major contributors: chronic systemic inflammation, hypercoagulable state, platelet activation \& oxidative stress. ${ }^{5}$ The persistent inflammatory state can trigger coagulation by promoting tissue-factor gene expression in endothelial cells. ${ }^{6}$ Hypoxia also could either reduce endothelial thrombomodulin expression or activate factor $X .^{7}$ Serum fibrinogen level also rises during COPD exacerbation. ${ }^{8}$ Platelet aggregability, as assessed in vivo by measuring 11-dehydro-thromboxane- $\beta-2$, the urinary metabolite of (TXA2) also shows elevated levels in hypoxemic COPD patients. ${ }^{9}$ Hydrogen peroxide in exhaled breath condensate, a marker of oxidative stress in lungs, has been found to be persistently elevated in COPD patients. ${ }^{10}$

Endothelial dysfunction, as characterized by increased levels of CRP, sICAM-1, IL-6, TNF- $\alpha$, endothelin-1, complement \& leptin as associated with COPD \& its exacerbation. ${ }^{11-13}$ Markers of hypercoagulation, Thrombin-Antithrombin III complex (TAT), fibrinopeptide A, \& plasminogen activator inhibitor type 1 (PAI-1) had shown definite correlation with COPD exacerbation. ${ }^{14}$ Prothrombin time (PT), activated Partial Thromboplastin Time (aPTT)) and von Willebrand Factor (VWF) are sensitive parameters of underlying hypercoagulable state. Fibrinogen is a marker of inflammation \& D-dimer is a marker of secondary fibrinolysis following thrombosis. ${ }^{15}$ These parameters, therefore, can be used as markers of procoagulant state in patients of COPD $\&$ its exacerbation. We did this study targeting the same.

\section{Methods}

It was an observational cross-sectional study which was carried out in the Department of Medicine, Maulana Azad Medical College, New Delhi after approval from institutional ethics committee. Study subjects were 30 in-hospital patients of COPD with acute exacerbation and 30 stable patients of diagnosed COPD (As per GOLD 2016) Patients having acquired thrombotic risk factors like diabetes, hypertension, coronary artery disease, chronic kidney disease, chronic liver disease, malignancy \& sepsis or any immunocompromised condition were excluded from the study. Also, patients who were on anticoagulant therapy, statins or antihypertensive medications were excluded. The recruited patients were subjected to detailed history, physical examination and severity assessment (based on spirometry using portable spirometer- spirolab II). All the patients were prescribed a standard treatment regimen for COPD management. Those in exacerbation were admitted for proper treatment and discharged after a minimum of 5 days of inpatient care in hospital.

Blood samples were drawn on two different occasions - On day 1 of admission \& on day 5 or later when the patients were clinically stable. For stable COPD patients only one sample was taken. Venous blood was collected into 3.8\% trisodium citrate tubes for assessment of PT, aPTT, VWF, fibrinogen and D-dimer. In this study, we considered P-value of $<0.05$ as statistically significant.

\section{Results and Observations}

Thirty patients each of COPD with acute exacerbation and stable COPD were enrolled in the study. Amidst the uniform distribution of study subjects in various age groups, most patients were in the age group of $41-70$ years corresponding to the peak age group of COPD population. $5 \%$ fell into $31-$ 40 years, $30 \%$ in $41-50$ years, $28.3 \%$ in $51-60$ years, $25 \%$ in $61-70$ years $\& 11.7 \%$ in $>70$ years age group. In this study, 47 patients were males $\& 13$ were females. The mean BMI of all patients was $25 \pm 5.3$. $33 \%$ of patients were having their $\mathrm{BMI}<23.5 \mathrm{~kg} / \mathrm{m}^{2}$.

After categorizing the patients based on severity of COPD using GOLD 2016, 4 patients were in GOLD stage II (moderate), 30 patients were in GOLD stage III (severe) \& 26 patients were in GOLD stage IV (very severe). Amongst the exacerbation group, patients fell equally into stage III \& IV. All patients were smokers with a smoking mean of $25 \pm 5.9$ pack years.

\section{Analysis of Coagulation Parameters and Prothrombotic Markers}

All the parameters like PT, aPTT, fibrinogen and vWF were obtained as quantitative values except D-dimer which was measured qualitatively and expressed as positive or negative. In COPD exacerbation group, out of 30 patients, 20 had negative and 10 had positive D-dimer value. Out of these 10 patients only 4 patients remained positive on follow up after minimum of 5 days of inpatient stabilization. On the other hand, only 5 patients out of thirty had positive result in stable COPD group. 
Table 1.Prothrombotic Markers in different stages of COPD at the time of exacerbation

\begin{tabular}{|c|c|c|c|c|}
\hline Prothrombotic Markers & GOLD status & $\mathbf{N}$ & Mean \pm S.D. & p-value \\
\hline \multirow{2}{*}{ PT } & Severe & 15 & $1.1010 \pm 0.7539$ & \multirow{2}{*}{0.08} \\
\hline & Very severe & 15 & $1.1741 \pm 0.1375$ & \\
\hline \multirow{2}{*}{ aPTT } & Severe & 15 & $1.1208 \pm 0.1804$ & \multirow{2}{*}{0.57} \\
\hline & Very severe & 15 & $1.1679 \pm 0.2643$ & \\
\hline \multirow{2}{*}{ vWF } & Severe & 15 & $147.45 \pm 14.23$ & \multirow{2}{*}{0.96} \\
\hline & Very severe & 15 & $147.70 \pm 16.61$ & \\
\hline \multirow{2}{*}{ Fibrinogen } & Severe & 15 & $373.08 \pm 152.63$ & \multirow{2}{*}{0.002} \\
\hline & Very severe & 15 & $609.10 \pm 228.58$ & \\
\hline
\end{tabular}

Table 2.Comparison between COPD exacerbation and Post stabilisation

\begin{tabular}{|c|c|c|c|}
\hline Prothrombotic markers & COPD exacerbation & Post Stabilisation & p-value \\
\hline PT & $1.13(1.09-1.18)$ & $1.02(1.00-1.03)$ & 0.011 \\
\hline aPTT & $1.14(1.06-1.22)$ & $1.03(1.01-1.05)$ & 0.027 \\
\hline VWF & $147.58(141.90-153.25)$ & $80.71(73.95-88.7)$ & $<0.001$ \\
\hline Fibrinogen & $491.09(406.86-575.31)$ & $267.45(233.93-300.97)$ & $<0.001$ \\
\hline
\end{tabular}

Table 3.Comparison between Exacerbation and Stable COPD group

\begin{tabular}{|c|c|c|c|}
\hline Prothrombotic markers & COPD exacerbation & Stable COPD & p-value \\
\hline PT & $1.13(1.09-1.18)$ & $1.09(1.00-1.18)$ & 0.51 \\
\hline aPTT & $1.14(1.06-1.22)$ & $1.14(1.07-1.20)$ & 0.99 \\
\hline vWF & $147.58(141.90-153.25)$ & $128.95(117.07-140.83)$ & 0.006 \\
\hline Fibrinogen & $491.09(406.86-575.31)$ & $426.86(373.08-480.63)$ & 0.28 \\
\hline
\end{tabular}

Table 4.Prothrombotic Markers in different stages of stable COPD patients

\begin{tabular}{|c|c|c|c|c|}
\hline Prothrombotic Marker & GOLD stage & $\mathbf{N}$ & Mean $\pm S . D$. & P-value \\
\hline \multirow{3}{*}{ PT } & Moderate & 4 & $1.04 \pm 0.56$ & \multirow{3}{*}{0.565} \\
\hline & Severe & 15 & $1.14 \pm 0.31$ & \\
\hline & Very severe & 11 & $1.04 \pm 0.09$ & \\
\hline \multirow{3}{*}{ aPTT } & Moderate & 4 & $1.01 \pm 0.038$ & \multirow{3}{*}{0.34} \\
\hline & Severe & 15 & $1.16 \pm 0.221$ & \\
\hline & Very severe & 11 & $1.15 \pm 0.127$ & \\
\hline \multirow{3}{*}{ vWF } & Moderate & 4 & $124.22 \pm 53.19$ & \multirow{3}{*}{0.079} \\
\hline & Severe & 15 & $117.90 \pm 28.91$ & \\
\hline & Very severe & 11 & $145.74 \pm 20.31$ & \\
\hline \multirow{3}{*}{ Fibrinogen } & Moderate & 4 & $411.77 \pm 111.25$ & \multirow{3}{*}{0.011} \\
\hline & Severe & 15 & $359.86 \pm 82.16$ & \\
\hline & Very severe & 11 & $523.70 \pm 172.72$ & \\
\hline
\end{tabular}

In patients of COPD with acute exacerbation ( $\mathrm{N}=30)$, stage wise distribution of prothrombotic markers (mean and standard deviation) is shown in Table 1. While comparing mean values using analysis of variance (ANNOVA), it was 
found to be statistically significant between stage III (severe) and IV (very severe) COPD for serum fibrinogen level only $(p=0.002)$.

In COPD exacerbation group, the mean value of fibrinogen was $491 \pm 225 \mathrm{mg} \%$, PT was $1.13 \pm 0.11$, aPTT was $1.14 \pm 0.22$ \& vWF was $147 \pm 15 \%$. When COPD exacerbation patients were stabilized after a minimum of 5 days of inpatient care, the mean values of fibrinogen, PT, aPTT \& vWF were $267 \pm 33.52 \mathrm{mg} \%, 1.02 \pm 0.02,1.03 \pm 0.02$ and $80.71 \pm 6.76 \%$ respectively (Table 2 ).

The values of PT, aPTT, vWF and fibrinogen were observed to be raised significantly as compared to these values after stabilization (day 5). This is in agreement with our hypothesis that COPD exacerbation is a prothrombotic state.

In stable COPD group ( $\mathrm{N}=30)$, however, the mean value of fibrinogen was $426 \pm 144 \mathrm{mg} \%$, PT was $1.09 \pm 0.22$, aPTT was $1.14 \pm 0.17 \&$ VWF was $128 \pm 31.8$. When we compared the values of coagulation parameters and prothrombotic markers between COPD exacerbation and stable COPD group patients enrolled in our study, only VWF was found to be significantly raised in exacerbation phase in comparison to the stable COPD group (Table 3). Although, the mean values of these parameters were much higher than the normal reference range even in these stable COPD patients. ${ }^{17}$

In patients of Stable COPD ( $\mathrm{N}=30)$, stage wise distribution of prothrombotic markers (mean and standard deviation) is shown in Table 4. While comparing mean values using analysis of variance (ANNOVA), it was found to be statistically significant between stage III (severe) and IV (very severe) COPD for Serum fibrinogen level only.

A significant difference was seen in the mean values of aPTT, vWF antigen and fibrinogen levels while comparing parameters between stable COPD group and post stabilization (day 5) group, as shown in Table 5.

Table 5.Comparison between post stabilization and stable COPD patients' group

\begin{tabular}{|c|c|c|c|}
\hline Prothrombotic markers & Stable COPD & Post stabilization & p-value \\
\hline PT & $1.0(1.00-1.18)$ & $1.02(1.00-1.03)$ & 0.162 \\
\hline aPTT & $1.14(1.07-1.20)$ & $1.03(1.01-1.05)$ & 0.029 \\
\hline VWF & $128.95(117.07-140.83)$ & $80.71(73.95-88.7)$ & $<0.001$ \\
\hline Fibrinogen & $426.86(373.08-480.63)$ & $267.45(233.93-300.97)$ & 0.001 \\
\hline
\end{tabular}
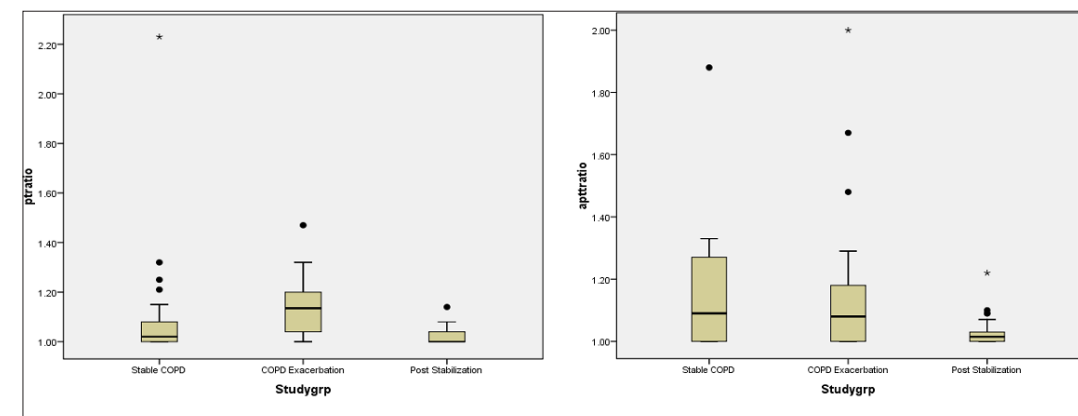

Figure 1(a)

Figure 1(b)

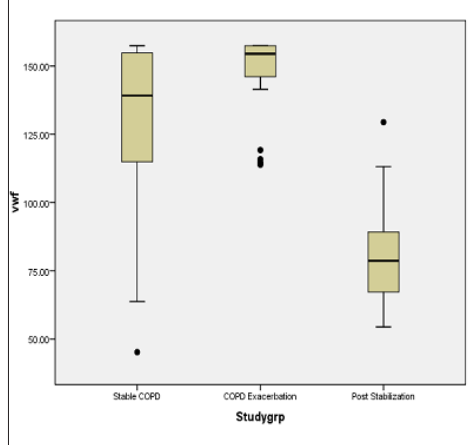

Figure 1(d)

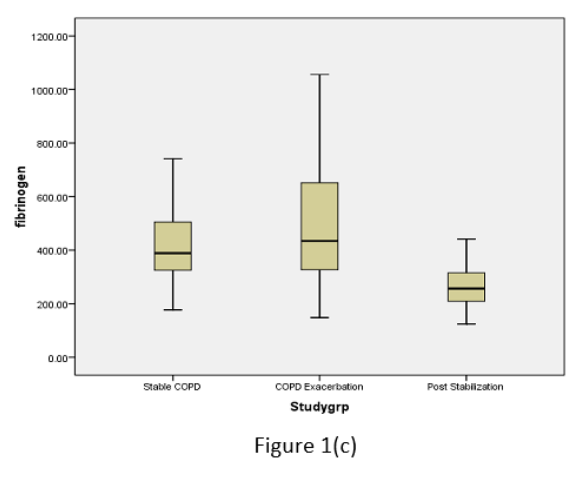

Diagrammatic representation of various parameters among different group of patients are shown below in Figure 1(a-d) (using Box-whisker plot)

Figure 1(a-d).Box Whisker plot showing comparison of various parameters among different study groups. [a: PT, b:aPTT, c: vWF, d: Fibrinogen] 


\section{Discussion}

This study was done to assess the prothrombotic state in COPD in various stages i.e., stable state, acute exacerbation and after its stabilization. There is paucity of data evaluating the relation between various stages of COPD with respect to various prothrombotic parameters like PT, aPTT, fibrinogen and vWF.

In our study, these parameters showed declining trends from exacerbation to post stabilization phase [PT $(p=0.001)$ $\& \operatorname{aPTT}(p=0.027)]$. In this study, the mean value of vWF declined after stabilization as compared to exacerbatory phase ( $p$-value<0.001). Also, stable COPD group had higher values than the normal reference range. In a recent study done by Mehmet Polati et al, ${ }^{15}$ vWF level was found to be the highest in acute exacerbation phase as compared to stable state, supporting our finding. Study conducted by Polosa et al showed $122 \%$ decline in vWF level from exacerbatory phase to the state of clinical stability. ${ }^{16}$

Fibrinogen, an acute phase reactant, plays a prominent role in clotting. The increase in its level, as it is associated with endothelial damage, has been found to be associated with the risk of cardiovascular disease. ${ }^{17}$ In our study also the mean value of fibrinogen showed a declining trend after stabilization as compared to exacerbatory state ( $p$-value $<0.001$ ). Out of the parameters studied, fibrinogen was the only parameter which showed graded increase with increasing severity of COPD. This finding is similar to the finding of the meta analysis where there was graded increase in fibrinogen level with increase in severity of COPD. ${ }^{18}$ Mehmet Polati et al, however, found that microalbuminuria, vWF and fibrinogen all were helpful in grading the severity of COPD exacerbation. ${ }^{15}$

D-dimer, the specific degradation product of cross-linked fibrin, was measured qualitatively in our study. It can be used as a molecular marker of hypercoagulation and secondary increased fibrinolytic activity. In this study, 10 patients had positive D-dimer value which declined to 4 patients in post stabilization phase $(p=0.008)$. These findings were also supported by other studies. ${ }^{19}$

This study provided evidence that acute exacerbation of COPD is associated with augmented prothrombotic state. The markers of coagulation such as PT, APTT and markers of prothrombotic state: fibrinogen, D-dimer and vWF were significantly raised in acute exacerbation which subsequently decreased on stabilization. The systemic inflammation during COPD exacerbation may be the cause of this prothrombotic state. These markers were also raised significantly in stable COPD patients as compared to post stabilization value. However, patients of COPD with acute exacerbation post stabilization had lesser degree of procoagulants than stable COPD patients. This can be explained by the fact that COPD is a chronic inflammatory disease in itself. Lesser degree of procoagulants in patients of COPD exacerbation post-stabilization might be attributable to the use of intravenous steroid therapy which decreases the systemic inflammation. While, in the stable COPD patients the systemic inflammation is ongoing and hence these markers had higher values than the normal population.

The elevated vWF, D-dimer and fibrinogen concentrations in the blood during acute exacerbation of COPD may precipitate thromboembolic events in these patients just as seen in ischemic heart disease. ${ }^{20,21}$ This is substantiated by deaths from pulmonary thromboembolism occuring in about $10 \%$ of patients admitted with acute exacerbation of COPD. ${ }^{22}$ The heightened procoagulant state during COPD exacerbation may predispose to venous thromboembolism and could, in principle, justify a general recommendation for anticoagulation and pharmacological thromboprophylaxis in these patients during exacerbation of their disease.

Present study has demonstrated the presence of hypercoagulable state in COPD patients. However, it has the limitation of small sample size. Further studies are needed to identify the exact mechanisms underlying the hypercoagulable state in COPD, and to determine whether anticoagulant therapy may be clinically useful in exacerbation of COPD. In our study, out of all parameters only vWF showed significant difference between exacerbation phase and stable phase of COPD ( $p<0.006)$. As the current study was not intended to prove VWF as a marker of exacerbation of COPD, further studies are required to clearly indicate whether VWF can be used as marker of acute exacerbation of COPD.

\section{Conclusion}

- $\quad$ COPD with acute exacerbation is a state of augmented prothrombotic state.

- On stabilization of acute exacerbation with treatment coagulation profile came to a level even lower than the patients in stable phase of COPD.

- $\quad$ VWF had been found to be significantly elevated in acute exacerbation phase of COPD than in stable phase.

\section{Abbreviation}

COPD: Chronic Obstructive Pulmonary Disease; PT: Prothrombin Time; PTT: Activated Partial Thromboplastin Time; VWF: Von Willebrand Factor; TNF: Tumour Necrosis Factor; LTs: Leukotrienes, Interleukins; CAD: Coronary Artery Disease; TX: Thromboxane; CRP: C-Reactive Protein; GOLD: Global Initiative for Chronic Obstructive Lung Disease.

\section{Declaration}

- $\quad$ Ethics Approval: This study was conducted only after approval from the institutional ethical committee 
Maulana Azad Medical College and after obtaining informed consent from all the patient participants.

- Funding: No organized funding source was used in study conduction.

- Acknowledgements: None

Conflict of Interest: None

References

1. Pauwels RA, Buist AS, Calverley PM, Jenkins CR, Hurd SS. Global strategy for the diagnosis, management, and prevention of chronic obstructive pulmonary disease. NHLBI/WHO Global Initiative for Chronic Obstructive Lung Disease (GOLD) workshop summary. Am J Respir Crit Care Med 2001; 163: 1256-76.

2. Wouters EF. Chronic obstructive pulmonary disease. 5: Systemic effects of COPD. Thorax 2002; 57: 1067-70

3. Zureik M, Benetos A, Neukirch C. Reduced pulmonary function is associated with central arterial stiffness in men. Am J Respir Crit Care Med 2001; 164: 2181-5.

4. Hansell AL, Walk JA, Soriano JB. What do chronic obstructive pulmonary disease patients die from? A multiple cause coding analysis. Eur Respir J 2003; 22: 809-14.

5. Pasceri V, Willerson JT, Yeh ET. Direct proinfl ammatory effect of C-reactive protein on human endothelial cells. Circulation 2000; 102: 2165-8.

6. Esmon CT. Does inflammation contribute to thrombotic events. Haemostasis 2000; 30(Suppl 2): 34-40.

7. Ogawa S, Shreeniwas R, Brett J. The effect of hypoxia on capillary endothelial cell function: modulation of barrier and coagulant function. Br J Haematol 1990; 75: 517-24.

8. Wedzicha JA, Seemungal TA, MacCallum PK. Acute exacerbations of chronic obstructive pulmonary disease are accompanied by elevations of plasma fibrinogen and serum IL-6 levels. Thromb Haemost 2000; 84: 210-15.

9. Patrono C, Patrignani P, Rocca B. Characterization of biochemical and functional effects of antiplatelet drugs as a key to their clinical development. Thromb Haemost 1995; 74: 396-400.

10. Nowak D, Kalucka S, Bialasiewicz P. Exhalation of $\mathrm{H} 2 \mathrm{O} 2$ and thiobarbituric acid reactive substances (TBARs) by healthy subjects. Free Radic Biol Med 2001; 30: 178-86.

11. Szmitko PE, Wang CH, Weisel RD, de Almeida JR, Anderson TJ, Verma S. New markers of inflammation and endothelial cell activation: part I. Circulation 2003; 108: 1917-23.

12. Fischetti F, Tedesco F. Cross-talk between the complement system and endothelial cells in physiologic conditions and in vascular diseases. Autoimmunity 2006; 39: 417-28.

13. Wedzicha JA, Syndercombe-Court D, Tan KC. Increased platelet aggregate formation in patients with chronic airflow obstruction and hypoxaemia. Thorax 1991; 46: 504-7.

14. Ashitani J, Mukae H, Arimura Y, Matsukura S. Elevated plasma procoagulant and fibrinolytic markers in patients with chronic obstructive pulmonary disease. Intern Med 2002; 41: 181-5.

15. Mehmet Polatli, Aysel C akir, Orhan Cildag. Microalbuminuria, von Willebrand factor and fibrinogen levels as markers of the severity in COPD exacerbation. J Thromb Thrombolysis 2008; 26: 97-102.

16. Polosa R, Malebra M, Cacciola RR, Morjaria JB, Maugeri C, Prosperini G. Effect of acute exacerbation on circulating endothelial, clotting and fibrinolytic markers in COPD patients. Internal emergency medicine 2011.

17. Kannel WB, Wolf PA, Castelli WP. Fibrinogen and risk of cardiovascular disease. JAMA 1987;258: 1183-6.

18. Bo Zhou, Shufang Liu, Danni He, Kundi Wang, Yunfeng Wamg, Ting Yang et al. Fibrinogen is a promising biomarker for chronic obstructive pulmonary disease: evidence from a meta-analysis. Biosci Rep 2020; 40(7): BSR20193542. DOI: 10.1042/BSR20193542.

19. Silva DR, Coelho AC, Gazzana MB, Menna Barreto SS, Knorst MM. D-dimer levels in stable COPD patients: a case-control study. COPD 2012; 9(4): 426-31.

20. Lowe GD, Yarnell JW, Sweetnam PM, Rumley A, Thomas HF, Elwood PC. Fibrin D-dimer, tissue plasminogen activator, plasminogen activator inhibitor, and the risk ofmajor ischaemic heart disease in the Caerphilly Study. Thromb Haemost 1998; 79(1): 129-133.

21. Rumley A, Lowe GD, Sweetnam PM, Yarnell JW, Ford RP. Factor VIII, von Willebrand factor and the risk of major ischaemic heart disease in the Caerphilly Heart Study. Br J Haematol 1999; 105(1): 110-6.

22. Groenewegen $\mathrm{KH}$, Schols AM, Wouters EF. Mortality and mortality-related factors after hospitalization for acute exacerbation of COPD. Chest 2003; 124 (2): 459-67. 